\title{
Managing an extensively drug-resistant tuberculosis outbreak: the public health face of the medal
}

\author{
To the Editor:
}

We read with interest the letter by EsPosito et al. [1], reporting on the clinical management of a paediatric case of extensively drug-resistant tuberculosis (XDR-TB) in Milan, Italy, who faced a life-threatening condition [2,3] that required access to an electronic Consilium [4]. The article is important as it describes a complex epidemic originated by a really difficult-to-treat case who had an intensive social life in a large city, thus making it necessary to perform a vast tracing of contacts, stratified into circles at different risk of exposure.

In the context of tuberculosis (TB) elimination [5-7], when TB incidence decreases and health services are obliged, based on cost-effectiveness considerations, to abandon the old-style vertical approach and integrate prevention, clinical and control functions, several difficulties gradually emerge [8,9]. First, a central authority, explicitly empowered to coordinate the outbreak management, is not generally available [5, 10]. Secondly, coordination between the different services involved (clinical and preventive medicine), between themselves and with local/regional/national health authorities, are usually not regulated. Last but not least, a trained focal point with a mandate to deal with media is usually missing [10]. A recent European Centre for Disease Prevention and Control (ECDC) survey clearly demonstrated the limited capacity of European Union countries in dealing with TB outbreaks and advocated for further public health research in this area [10].

Milan and the Lombardy region (the most populated region in Italy), due to specific epidemiological features (large population, important migrant community, TB incidence higher than the national rate, previous experience in managing TB outbreaks, and historical leadership in TB control through the establishment of a regional coordination group) has established a permanent task force to deal with outbreaks/micro-epidemics [8]. The group includes the Regional Office for Public Health (Milan, Italy), the Reference TB Centre and Laboratory (Milan), plus the public health districts and clinicians involved in the various outbreaks. In the present case, the National Centre for Molecular Surveillance of Mycobacterium tuberculosis (Milan) performed the extra-regional laboratory investigations, and Stop TB Italy (a local nongovernmental organisation member of the Stop TB Partnership) was involved in the counselling of contacts and communication with the press.

We will describe here how the Milan XDR-TB outbreak was managed, from the public health perspective, to complete the information provided in the letter by Esposito et al. [1], and to quantify by cost-description its direct costs.

All activities performed in order to investigate the index and secondary cases [1], to provide educational and counselling services to cases (the majority being children), relatives, healthcare workers and other individuals involved, to perform contact-tracing and to deal with the media are reported in table 1.

The average gross hourly cost (€28.10), as derived from official Italian figures for 2013 [11], has been applied to all direct costs related to activities performed by heathcare workers involving relatives employed in the different circles evaluated during contact-tracing, and any other person involved. In addition, the costs of performing microbiological examinations (sputum smears, cultures, drug susceptibility testing, etc.) and diagnosis for latent infection (Mantoux tests and interferon- $\gamma$ release assays (IGRAs)) have been also added (table 1). In total, five cases were involved in the outbreak. Of these, two were microbiologically confirmed: the index case (an Italian 12-yr-old male with pulmonary and laryngeal TB) and one of his classmates (a Romanian male of the same age, with pleural effusion). Three were not confirmed: the two younger siblings of the index case (with minimal pulmonary lesions) and another classmate (a female from Morocco who was independently diagnosed with initial TB and successfully treated with a standard regimen in another hospital). Contact tracing has been organised in five different circles, and included screening of 320 individuals. Out of the 55 who were positive for the Mantoux test (Tubertest 5 UT; 
TABLE 1 Costs of activities performed in order to investigate the index and secondary cases in the extensively drug-resistant TB outbreak in Milan, Italy

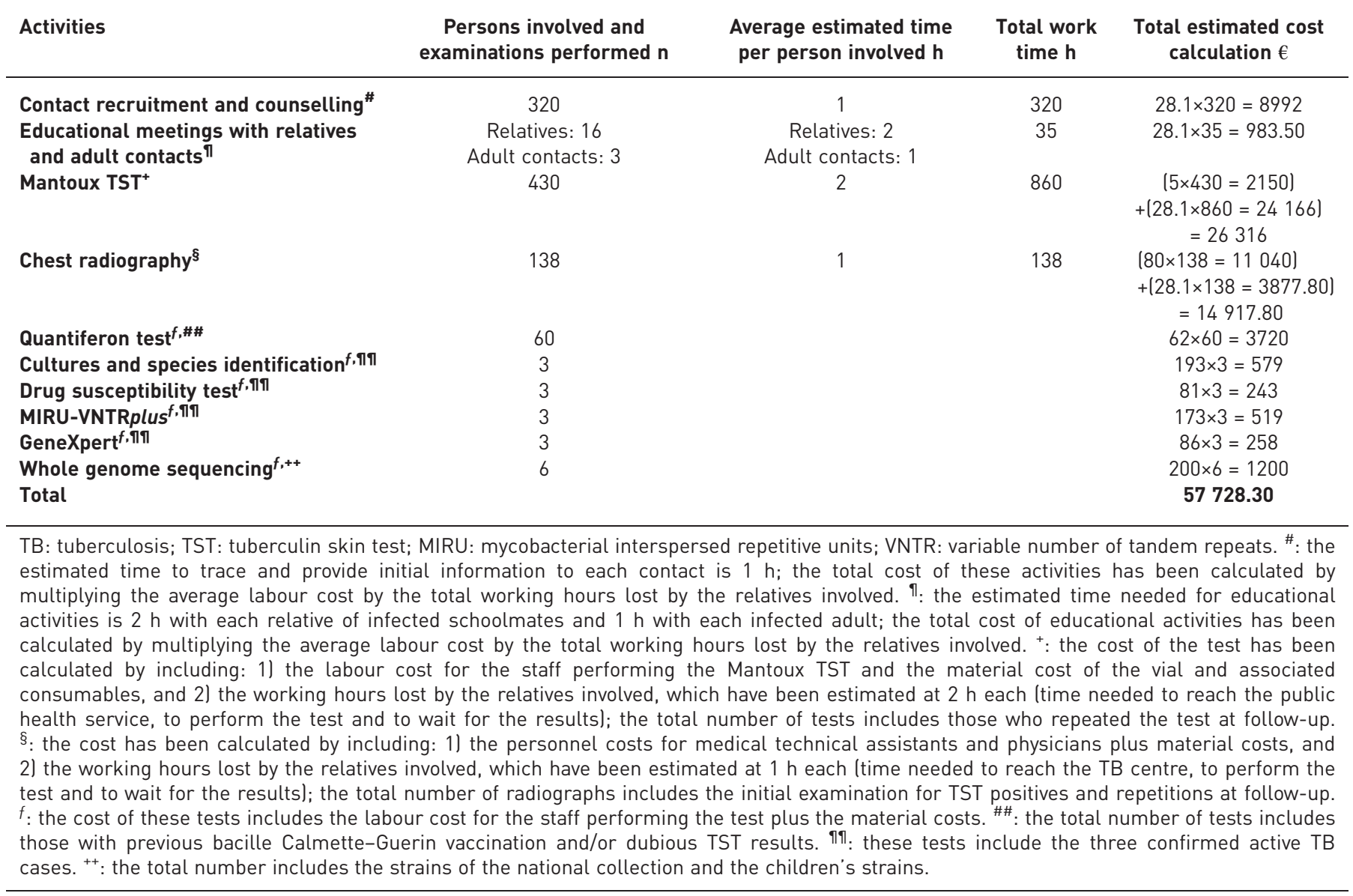

Sanofi Pasteur MSD, Paris, France), 36 underwent confirmation with the Quantiferon test (Qiagen, Valencia, CA, USA) and eight were positive. Patients with negative Mantoux or IGRA were retested after 2 months, while those who were positive started a follow-up with controls at 3, 6, 12 and 24 months at the Reference TB Centre.

Molecular epidemiology on 24 samples obtained from the culture-positive children unexpectedly demonstrated that the two strains isolated were identical to two strains previously isolated in Italy. The Regional Office for Public Health requested whole genome sequencing (WGS) to achieve a higher level of discrimination. WGS showed almost complete identity between one of the two strains of the national collection and the children's strains.

Overall, the direct costs of similar epidemics are relevant. The figures presented here are conservative because: 1) they do not consider the fact that the hourly cost of healthcare staff (medical doctors in particular) and of several of the relatives involved are higher than the average national cost; 2) other direct costs (transportation, etc.) were not added; and 3) indirect costs (patients' and relatives' stress and suffering, and loss of productivity related to the working hours lost, etc.), as well as the costs of admission and treatment, have not been calculated.

This experience demonstrates that: 1) TB outbreaks absorb important resources that might otherwise be devoted to other health-related activities; 2) correct clinical management and adequate coordination among prevention, clinical and control health services might prevent TB outbreak occurrence and/or minimise their effects; 3 ) in large cities, a stable mechanism of coordination is advisable to ensure preparedness to deal adequately with TB outbreaks [9]; and 4) national molecular surveillance is an important tool for outbreak management. 

essential to minimise effects http://ow.ly/B5Mqh

Luigi R. Codecasa ${ }^{1}$, Giorgio Ciconali ${ }^{2}$, Ester Mazzola ${ }^{3}$, Maurizio Ferrarese ${ }^{1}$, Daniela Cirillo ${ }^{4}$, Emanuele Borroni $^{4}$, Giovanni P. Gesu ${ }^{3}$, Jean-Pierre Zellweger ${ }^{5}$, Rosella Centis ${ }^{6}$ and Marino Faccini ${ }^{2}$

${ }^{1}$ Regional TB Reference Centre, Villa Marelli Institute/Niguarda Ca' Granda Hospital, Milan, Italy. ${ }^{2}$ Prevention Dept, ASL Milano, Milan, Italy. ${ }^{3}$ Regional TB Reference Laboratory, Niguarda Ca' Granda Hospital, Milan, Italy. ${ }^{4} \mathrm{~TB}$ Supranational Reference Laboratory, IRCCS San Raffaele Scientific Institute, Milan, Italy. ${ }^{5}$ TB Competence Center, Swiss Lung Association, Bern, Switzerland. ${ }^{6}$ World Health Organization Collaborating Centre for Tuberculosis and Lung Diseases, Fondazione S. Maugeri, Care and Research Institute, Tradate, Italy.

Correspondence: Luigi R. Codecasa, Regional TB Reference Centre, Villa Marelli Institute/Niguarda Ca' Granda Hospital, Viale Zara 81, 20159 Milan, Italy. E-mail: luigi.codecasa@ospedaleniguarda.it

Received: July 232014 | Accepted: July 282014

Conflict of interest: None declared.

Acknowledgements: The authors would like to acknowledge the following co-investigators: Susanna Esposito and Samantha Bosis (Fondazione IRCCS Ca' Granda Ospedale Maggiore Policlinico, Milan, Italy); Elia Stupka, Davide Cittaro and Paolo Miotto (IRCCS San Raffaele Scientific Institute, Milan Italy); Stefan Niemann and Thomas Khol (Molecular Mycobacteriology, Borstel, Germany). The authors would also like to thank Maria Gramegna, Alessandra Piatti and Liliana Coppola (General Health Direction, Lombardy Region, Milan, Italy) and all the Public Health Nurses of the Milan 3rd Public Health District and of the TB Reference Centre who participated in the management of the Milan outbreak.

\section{References}

1 Esposito S, D'Ambrosio L, Tadolini M, et al. ERS/WHO Tuberculosis Consilium assistance with extensively drug-resistant tuberculosis management in a child: case study of compassionate delamanid use. Eur Respir J 2014; 44: 811-815.

2 Falzon D, Gandhi N, Migliori GB, et al. Resistance to fluoroquinolones and second-line injectable drugs: impact on multidrug-resistant TB outcomes. Eur Respir J 2013; 42: 156-168.

3 Migliori GB, Sotgiu G, Gandhi NR, et al. Drug resistance beyond extensively drug-resistant tuberculosis: individual patient data meta-analysis. Eur Respir J 2013; 42: 169-179.

4 D'Ambrosio L, Tadolini M, Dupasquier S, et al. ERS/WHO Tuberculosis Consilium: reporting of the initial 10 cases. Eur Respir J 2014; 43: 286-289.

5 D’Ambrosio L, Dara M, Tadolini M, et al. Tuberculosis elimination: theory and practice in Europe. Eur Respir J 2014; 43: 1410-1420

6 Voniatis C, Migliori GB, Voniatis M, et al. Tuberculosis elimination: dream or reality? The case of Cyprus. Eur Respir J 2014; 44: 543-546.

7 Diel R, Loddenkemper R, Zellweger JP, et al. Old ideas to innovate tuberculosis control: preventive treatment to achieve elimination. Eur Respir J 2013; 42: 785-801.

8 Faccini M, Codecasa LR, Ciconali G, et al. Tuberculosis outbreak in a primary school, Milan, Italy. Emerg Infect Dis 2013; 19: 485-487.

9 Van Hest NA, Aldridge RW, de Vries G, et al. Tuberculosis control in big cities and urban risk groups in the European Union: a consensus statement. Euro Surveill 2014; 19: 20728

10 Migliori GB, Centis R, D'Ambrosio L, et al. Impact and management of TB childhood outbreaks in EU/EEA. Eur Respir J 2013; 42: Suppl. 57, 3532

11 Eurostat. Labour cost levels. http://appsso.eurostat.ec.europa.eu/nui/show.do?dataset=lc_lci_lev\&lang=enDate last updated: April 25, 2014.

\section{ERS/WHO Tuberculosis Consilium assistance in extensively drug-resistant tuberculosis}

To the Editor:

We read with interest the article by EsPosito et al. [1], whereby they emphasised the role of the European Respiratory Society (ERS)/World Health Organization (WHO) Tuberculosis (TB) Consilium [2-4] in the management of an extensively drug-resistant (XDR)-TB case. The creation of this e-platform brings 